\title{
Observation of Aging Effect on One Step Direct Injection Flame Synthesized Nano Alumina
}

\author{
P.Kathirvel \\ \{pkv.phy@psgtech.ac.in\} \\ GRD Centre for Materials Research, Department of Physics, PSG College of Technology, Coimbatore \\ 641004, Tamil Nadu, India.
}

\begin{abstract}
The nano alumina particles were produced by instant and continuous one step Direct Injection Flame Synthesis (DIFS). The synthesized nanoparticles were highly crystalline with orthorhombic size of crystal structure belonging to the space group of P222. The nano alumina particle has a crystallite $36 \mathrm{~nm}$ and the particle radius is calculated to be 24 $\mathrm{nm}$. The synthesized nano alumina particle exhibits a spherical morphology with different radius. The particles were subjected to controlled aging process and the formation of encapsulation layer is reported. The thickness of the encapsulation layer is found to be $\sim 4.5$ $\mathrm{nm}$.
\end{abstract}

Keywords: DIFS, P222, powder X-ray Diffraction

\section{Introduction}

Nano alumina (Al2O3) has found its application in the transistors, optoelectronic devices, composites, fillers, batteries and other high temperature applications owing to its physical and chemical stability at elevated temperatures, insulating properties and its excellent control over its size and morphology by economical synthesis methods[1][2][3]. This technologically important material can be synthesized by various routes including Flame synthesis, Hydrothermal, Chemical Vapor Deposition methods[1,4,5]. For this study the flame synthesis method has been chosen due to its ease synthesis process and its control over size and stoichiometry[6]. The demand for nano alumina is increasing over year continuously and a study on highly valued nano alumina on safe storage and its shelf life period is essential. In this study, the alumina nanoparticles aging effect has been investigated and reported.

\section{Methods and Materials}

Aluminum ( $\mathrm{Al}$ ) metal powder has been purchased from SRL chem., India with a mesh size of $74 \mu \mathrm{m}$ is chosen as the reactant material for DIFS. The experimental setup and method is already reported in our previous experiment[4]. The Al metal dust is directly fed into the neutral oxyacetylene flame white cone. This results in the spontaneous combustion and oxidation of $\mathrm{Al}$ 
metal particles into nano alumina which evolves as a white smoke from the neutral oxyacetylene flame. The resulting soot formation is collected and used for further studies. The sample collected is filled up to $20 \%$ volume of the air tight container for controlled aging observation.

To identify and understand the crystalline nature of the samples, they were subjected to the powder X-ray Diffraction (PXRD) studies carried out by Empyrean, Malvern Panalytical instrument using the $\mathrm{Cu}$ source $(\lambda=0.154 \mathrm{~nm})$. To analyze the composition, structural nature, size and fine morphology of the prepared sample they were dispersed in absolute ethanol and transferred onto the copper grid followed by atmospheric drying for the Energy Dispersive X -Ray Analysis (EDAX) analysis, Selected Area Electron Diffraction (SAED) and High Resolution Transmission Electron Microscope (HRTEM) studies which were carried out using JEOL JEM 2100 .

\section{Results and Discussion}
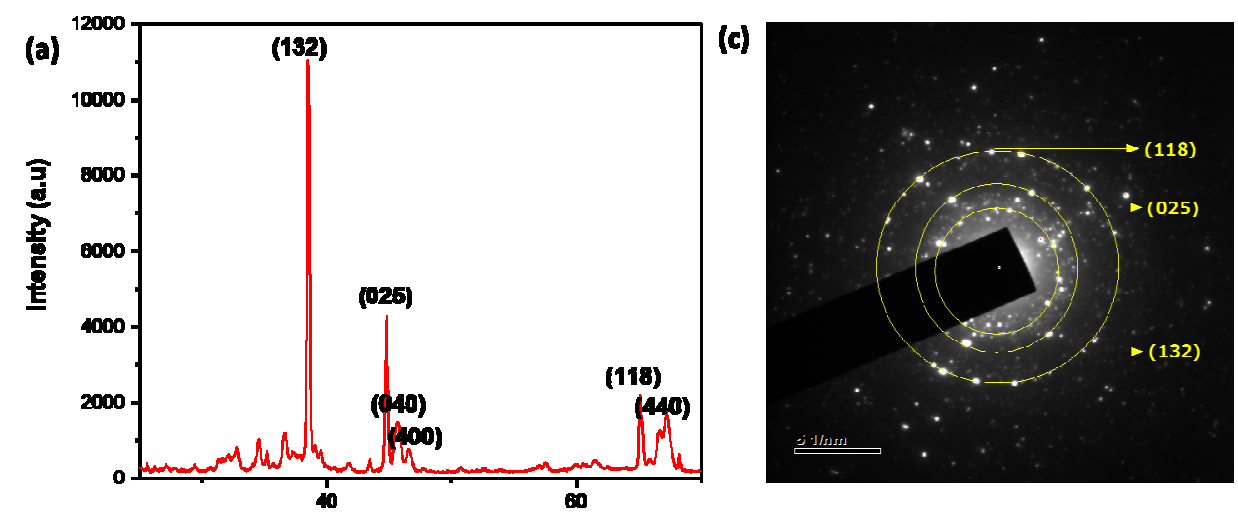

(b)
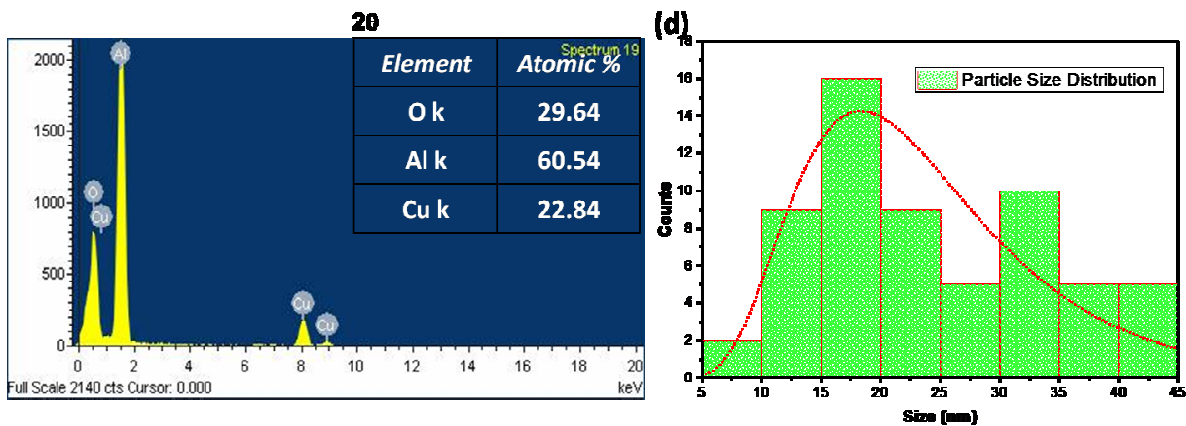

Fig 1. (a) PXRD pattern of nano alumina. (b) EDAX spectrum of nano alumina particles (insert: elemental composition). (c) SAED pattern of nano alumina particles. (d) Particle size distribution fitted with lognormal distribution. 
The powder X-ray diffractogram (Figure 1.a) shows peaks located at $38^{\circ} 49^{\prime \prime}, 44^{\circ} 74^{\prime}$, $45^{\circ} 42^{\prime}, 45^{\circ} 69^{\prime}, 65^{\circ} 10^{\prime}, 66^{\circ} 53^{\prime}$ and $67^{\circ} 17^{\prime}$ corresponding to the plane (132), (025), (040), (400), (118), (440) and (046) respectively. The peaks were sharp and intense indicating the good crystalline quality of the prepared sample. The peaks align with the standard JCPDS card no: 461215 confirming the formation of highly crystalline orthorhombic alumina belonging to the space group P222. The relative intensity of the planes does not match with the standard data. The most intense peak is found along (132) contrary to the (046) plane. Other planes relative intensity does not match with the standard data and the predominant growth plane confirmed by HRTEM is along (132) plane. Using the Debye - Scherrer equation (1), the average crystallite size (D) of the particle was found to be $36 \mathrm{~nm}$ and the dislocation density $(\delta)$ was found to be $7.71 \times 1014$ lines $\mathrm{m}$ 2 using equation (2)

$$
\mathbf{D}=\frac{0.9 \lambda}{\beta \cos \theta}
$$

$$
\delta=\frac{1}{D^{2}}
$$

where $\lambda$ is the wavelength of the X-ray source, $\theta$ is the peak position and $\beta$ is the full width half maximum of the peak. No other major peaks were observed in the PXRD indicating the phase purity of the sample which is further supported by the EDAX spectrum (Figure 1.b) which shows only the peaks belonging to the aluminum, oxygen and copper. The copper peak arises from the copper grid used for supporting the sample during EDAX study. The SAED pattern (Figure 1.c) shows circularly scattered dot pattern unique to powder nanoparticles and shows only the planes of orthorhombic alumina which confirms the formation of nano alumina. 
(a)

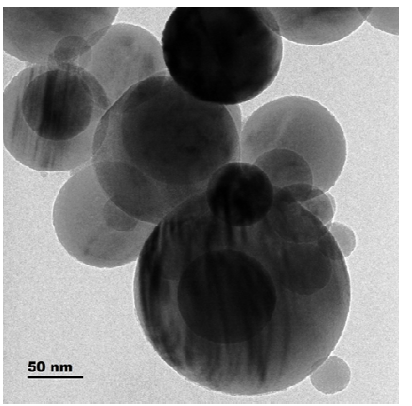

(b)

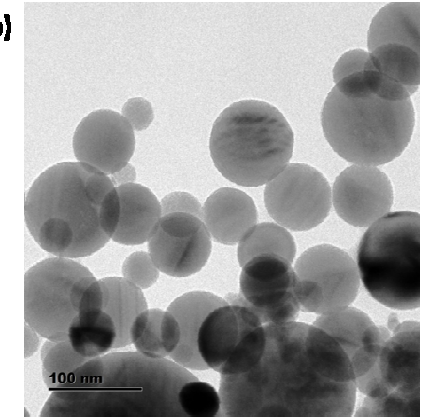

(c)

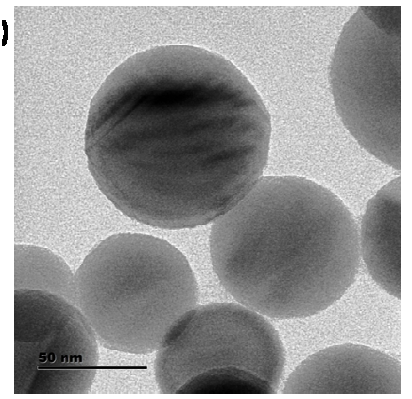

(d)

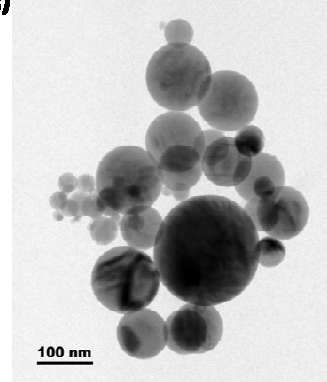

Figure 2. (a-d) HRTEM images of freshly prepared spherical nano alumina particles without any encapsulation layer.

HRTEM studies (Figure 2.a-d) reveal the spherical morphology of nano alumina. The average radius of the nano alumina is found to be $24 \mathrm{~nm}$ (Figure 1.d). Assuming the nano alumina to be a perfect sphere average surface area and volume of a particle is calculated using the basic geometry equation and found to be $8.36 \mu \mathrm{m} 2$ and $86.73 \mu \mathrm{m} 3$ respectively. The surface to volume ratio is found to be $0.9 / \mathrm{nm}$. The nano alumina shows slight agglomeration of adjacent particles which is a signature of flame synthesis[7,8]. 
(a)

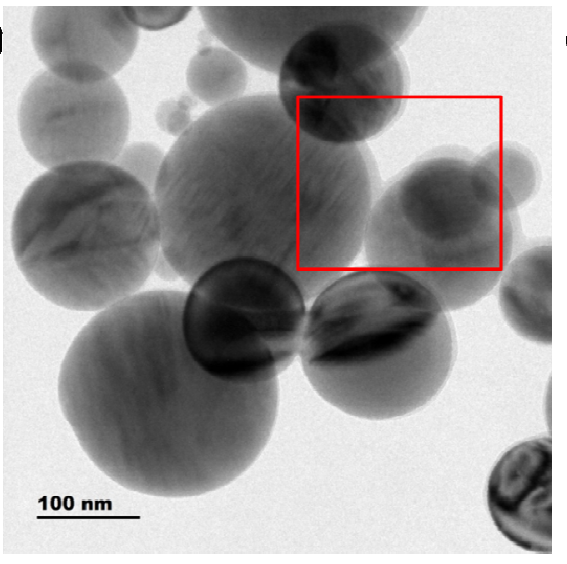

(c)

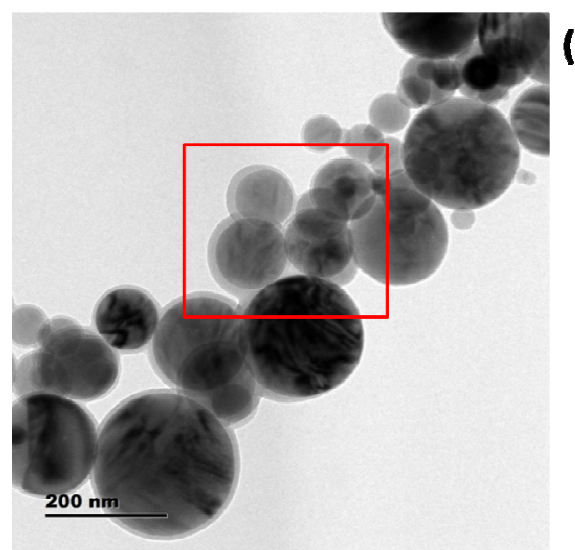

(b)

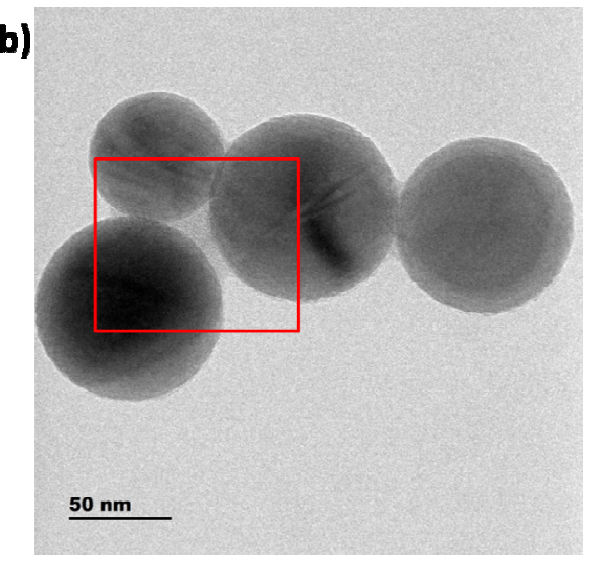

(d)

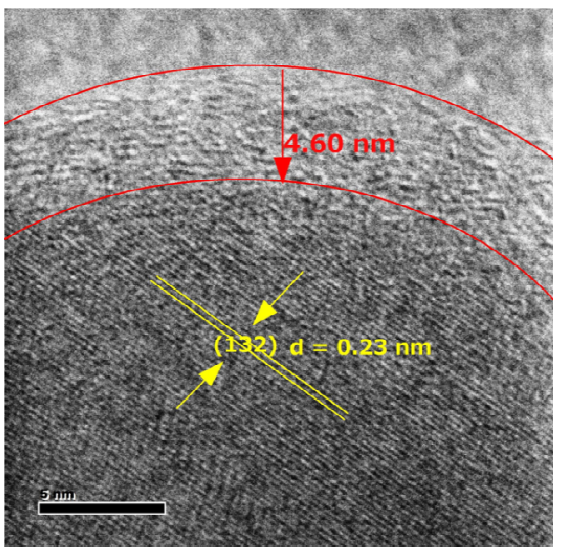

Fig 3. (a) Encapsulation layer over nano alumina due to aging effect. (b-c) Growth of encapsulation layer onto adjacent particles. (d) HRTEM image of encapsulation layer.

The freshly sample is devoid of layer formation on the outer surface but after the prolonged storage of the nano alumina there forms a nanolayer (Figure 4.a-c) with the average thickness of 4 to $5 \mathrm{~nm}$ around the particle (Figure 4.d). The thickness of the nanolayer is found to be uniform for a single particle but varies for different particles. This shows that the exposure of the nano alumina is different for each particle. This variation may cause due to the packing condition, air pockets between the particles and environmental temperature differences. The exact origin in these differences is unclear but we believe the surface level defects plays a significant role in attracting the atmospheric species to form a layer around it. Other important observation is that after prolonged storage the nanolayer on the alumina surface begins to form contact with other adjacent particles and develops further to encapsulate the total particle system and results in agglomeration. 


\section{Conclusion}

This investigation shows the synthesis and formation of highly crystalline orthorhombic structured nano alumina with a mean radius of $24 \mathrm{~nm}$. The formation of aging effect was confirmed after prolonged storage (6 months) of the synthesized nano alumina by the direct observation of encapsulated layer around the particle with a average thickness of $4-5 \mathrm{~nm}$. After further aging the encapsulated layer forms contact with adjacaent particle to form a agglomerated particle system. Overall, for the prolonged storage of nano alumina particles, inert atmosphere or vacuum environment is favored for pristine condition.

\section{References}

[1]. L. Fu, A. Huang, H. Gu, H. Ni, Elsevier Ltd and Techna Group S.r.1., (2018).

[2]. M. Shojaie-Bahaabad, E. Taheri-Nassaj, Mater. Lett. 62 3364-3366 (2008).

[3]. E.A. Trusova, D.D. Titov, A.N. Kirichenko, M.Y. Zorin, New J. Chem. 44 9046-9052(2020).

[4]. P. Kathirvel, J. Chandrasekaran, D. Manoharan, S. Kumar, 590 341-345(2014).

[5]. P.S. Behera, R. Sarkar, S. Bhattacharyya, NPG Asia Mater. 10-16(2009).

[6]. K. Karthick, P. Kathirvel, R. Marnadu, S. Chakravarty, M. Shkir, Phys. B Condens. Matter. 612 412971(2021).

[7]. E. Ramasamy, P. Kathirvel, S. Kumar, K. Suresh, G. Veerappan, MRS Commun. $7862-866$ (2017).

[8]. P. Kathirvel, J. Chandrasekaran, D. Manoharan, S. Kumar, Ceram. Int. 39 5321-5325 (2013). 